\title{
Automated Mesiodens Classification System Using Deep Learning on Panoramic Radiographs of Children
}

\author{
Younghyun Ahn ${ }^{1,+}$, Jae Joon Hwang $2,3,+\left(\mathbb{D}\right.$, Yun-Hoa Jung ${ }^{2,3}$, Taesung Jeong ${ }^{1,3}$ and Jonghyun Shin $1,3, * \mathbb{C}$ \\ 1 Department of Pediatric Dentistry, School of Dentistry, Pusan National University, Yangsan 50612, Korea; \\ ahn7750@pusan.ac.kr (Y.A.); tsjeong@pusan.ac.kr (T.J.) \\ 2 Department of Oral and Maxillofacial Radiology, School of Dentistry, Pusan National University, \\ Yangsan 50612, Korea; softdent@pusan.ac.kr (J.J.H.); yhjung@pusan.ac.kr (Y.-H.J.) \\ 3 Dental and Life Science Institute \& Dental Research Institute, School of Dentistry, Pusan National University, \\ Yangsan 50612, Korea \\ * Correspondence: jonghyuns@pusan.ac.kr; Tel.: +82-55-360-5183 \\ + These authors contributed equally to this work.
}

check for updates

Citation: Ahn, Y.; Hwang, J.J.; Jung, Y.-H.; Jeong, T.; Shin, J. Automated Mesiodens Classification System Using Deep Learning on Panoramic Radiographs of Children. Diagnostics 2021, 11, 1477. https://doi.org/ 10.3390/diagnostics11081477

Academic Editor: Hee-Cheol Kim

Received: 25 July 2021

Accepted: 12 August 2021

Published: 15 August 2021

Publisher's Note: MDPI stays neutral with regard to jurisdictional claims in published maps and institutional affiliations.

Copyright: (c) 2021 by the authors. Licensee MDPI, Basel, Switzerland. This article is an open access article distributed under the terms and conditions of the Creative Commons Attribution (CC BY) license (https:// creativecommons.org/licenses/by/ $4.0 /)$.

\begin{abstract}
In this study, we aimed to develop and evaluate the performance of deep-learning models that automatically classify mesiodens in primary or mixed dentition panoramic radiographs. Panoramic radiographs of 550 patients with mesiodens and 550 patients without mesiodens were used. Primary or mixed dentition patients were included. SqueezeNet, ResNet-18, ResNet-101, and Inception-ResNet-V2 were each used to create deep-learning models. The accuracy, precision, recall, and F1 score of ResNet-101 and Inception-ResNet-V2 were higher than 90\%. SqueezeNet exhibited relatively inferior results. In addition, we attempted to visualize the models using a class activation map. In images with mesiodens, the deep-learning models focused on the actual locations of the mesiodens in many cases. Deep-learning technologies may help clinicians with insufficient clinical experience in more accurate and faster diagnosis.
\end{abstract}

Keywords: mesiodens; artificial intelligence; deep learning; convolutional neural networks

\section{Introduction}

Supernumerary teeth are defined as the teeth that exceed the normal number of teeth. The cause of excessive number of teeth has not been clearly identified, and both genetic and environmental factors are presumed to be involved. Supernumerary teeth are most common in the midline of maxilla and are called mesiodens [1]. The prevalence of mesiodens has been reported to be $0.1-7.0 \%$ [2].

Mesiodens can cause various complications, such as dentigerous cysts, resorption of the roots of adjacent permanent teeth, eruption disorders of the maxillary incisors, diastema, and crowding. If inverted mesiodens are not detected early and left neglected, these may move towards the nasal cavity, increasing the difficulty in performing the surgical operation [3]. Therefore, to prevent such complications, it is imperative to detect the mesiodens in advance, and to extract them at an appropriate time.

If cone-beam computed tomography (CBCT) is used for the diagnosis of mesiodens, the precise shape and comprehensive location information of the impacted mesiodens can be easily identified in three dimensions [4]. However, children are more sensitive to radiation than adults are, and when radiographs of children are taken under the same conditions as those for adults, the risk of exposure is high [5]. For this reason, CBCT cannot be performed on a regular basis. It is necessary to evaluate the justification of exposure of radiation on children before using CBCT [6]. However, panoramic radiographs provide considerable diagnostic information within the jaw with a single radiograph and are often used in dentistry because they offer the advantage of relatively low radiation exposures, compared to those with СBCT [7]. Even in children, panoramic radiographs 
are often taken for evaluating the stage of tooth development and dental caries. However, the panoramic radiographs of patients with mixed dentition are complicated owing to the mixture of primary and successive permanent teeth; thus, there are several factors that dentists must carefully consider. Additionally, owing to the difference in size of the maxillary arch between children and adults, the image of the upper anterior region of a child is not accurately included in the focal trough [4]. Owing to these difficulties, dentists unfamiliar with mixed dentition panoramic radiographs may miss important diagnostic information [8].

Currently, the deep convolutional neural network (DCNN) technology is being actively applied in the field of dental imaging [9]. Artificial intelligence has been reported to excellently judge various diseases in the oral and maxillofacial areas [10-13]. With the introduction of class activation map technology, the black-box effect of deep-learning models has been overcome, and judgments can be visualized [14]. However, only a few studies have used artificial intelligence for diagnosing mesiodens, and each study on patients with mixed dentition has a limitation: only a single deep-learning model has been used $[15,16]$. To the best of our knowledge, there has not been any study that has visualized the decision of the deep-learning model using the class activation map to locate the mesiodens.

Therefore, this study aimed to use several deep-learning networks to create models that automatically detect mesiodens in panoramic radiographs of children with mixed dentition, evaluate the performance of each model, and visualize the model using the class activation map.

\section{Materials and Methods}

\subsection{Ethics Statement}

This study was conducted with the approval of the Institutional Review Board (IRB) of Pusan National University Dental Hospital (IRB No.: PNUDH-2020-006).

\subsection{Subjects}

This study was conducted on panoramic and CBCT radiographs of Pusan National University Dental Hospital patients from January 2013 to January 2020. Patients who were diagnosed with one or more mesiodens via $\mathrm{CBCT}$ were selected as the experimental group. As controls, dental age-matched patients without mesiodens were selected from the same database (Table 1). CBCT (Pax-Zenith3D; Vatech Co., Ltd., Hwaseong, Korea) was performed with the following scanning parameters: $105 \mathrm{kVp}, 4 \mathrm{~mA}, 24 \mathrm{~s}$, voxel size of $0.2 \mathrm{~mm}$, and field of view of $20 \times 19 \mathrm{~cm}$. All patients included in this study were in Hellman's dental developmental stages IIA, IIC, and IIIA [17]. Panoramic radiographs, which were difficult to interpret owing to severe distortions of images, and patients with developmental tooth disorders or orthodontic treatment were excluded. Panoramic radiographs of 550 patients in the experimental group and 550 patients in the control group were used in the study.

\subsection{Methods}

\subsubsection{Data Preprocessing}

All panoramic radiographs were taken using a Proline XC machine (Planmeca Co., Helsinki, Finland) and downloaded as JPEG files ( $2943 \times 1435$ pixels).

To increase the mesiodens detection accuracy of the deep-learning models, the anterior region was set as the region of interest (ROI). Therefore, one dentist manually cropped the images from the panoramic radiographs from the right to left end of both the maxillary permanent canine germs horizontally, and from the uppermost point of both maxillary permanent canine tooth germs to the mandibular anterior alveolar bone level vertically. The images were then saved as JPEG files (Figure 1). 
Table 1. Demographic data of subjects in this study.

\begin{tabular}{lcc}
\hline Characteristics & $\begin{array}{c}\text { Patients without Mesiodens } \\
(\boldsymbol{n}=\mathbf{5 5 0 )}\end{array}$ & $\begin{array}{c}\text { Patients with Mesiodens } \\
(\boldsymbol{n}=\mathbf{5 5 0 )}\end{array}$ \\
\hline $\begin{array}{l}\text { Mean Age (SD) } \\
\text { Sex }\end{array}$ & $7.2(1.3)$ & $6.8(1.0)$ \\
Female & 253 & 126 \\
Male & 297 & 424 \\
Hellman's stages & & \\
IIA $^{1}$ & 130 & 130 \\
IIC $^{2}$ & 385 & 385 \\
IIIA $^{3}$ & 35 & 35 \\
\hline
\end{tabular}

${ }^{1}$ IIA: Completion of primary occlusion, ${ }^{2}$ IIC: Eruptive phase of permanent first molars or incisors, ${ }^{3}$ IIIA: Eruption of permanent first molars or incisors completed.
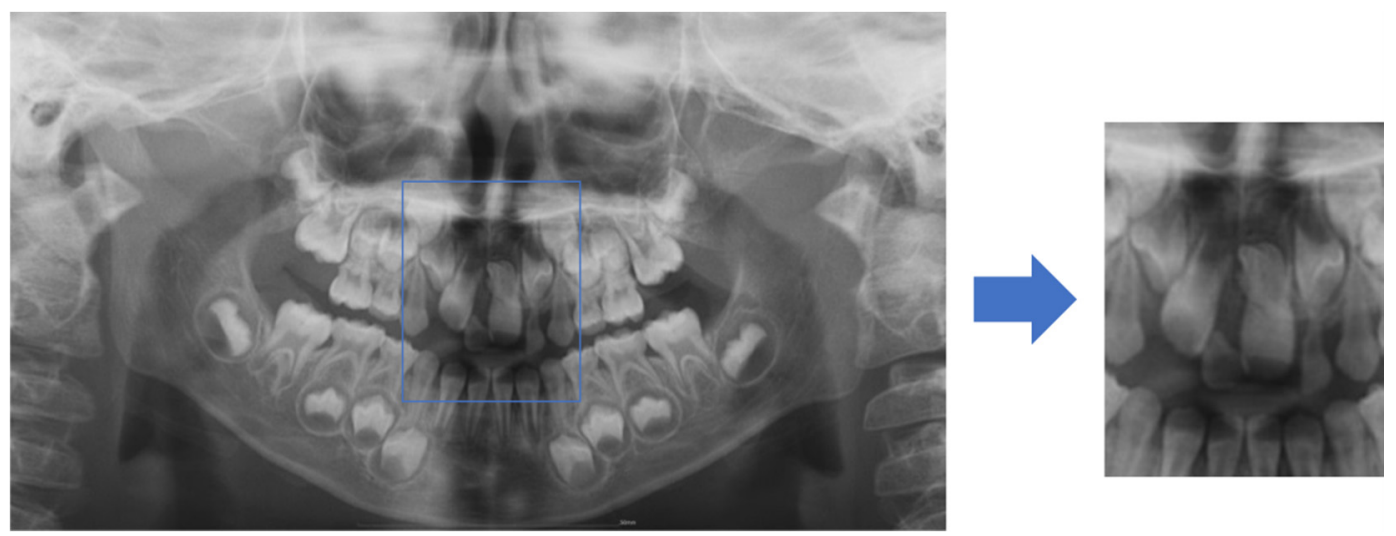

Figure 1. Region of interest (ROI). Images were cropped, as shown in blue box, based on the distal and uppermost points of both permanent canine tooth germs and mandibular anterior alveolar bone level.

\subsubsection{Data Classification}

The 1100 pre-processed images were divided into two groups. Dataset 1 was used to train the network and validate the performance of the trained model, and it consisted of 1000 images (500 images from the experimental group and 500 images from the control group). The remaining 100 images (50 images from the experimental group and 50 images from the control group) formed dataset 2 to compare the abilities of the deep-learning models and the human group to classify mesiodens.

\subsubsection{Network Pre-Training}

SqueezeNet, ResNet-18, ResNet-101, and Inception-ResNet-V2 networks were used for classifying mesiodens. Since all the networks were pre-trained using over a million images through the ImageNet database, it was possible to learn the rich features of various images. The basic properties of the network used in the study are presented in Table 2.

Table 2. Properties of pre-trained convolutional neural networks (CNNs).

\begin{tabular}{ccccc}
\hline Network Model & Depth & Size (MB) & $\begin{array}{c}\text { Parameter } \\
\text { (Millions) }\end{array}$ & Input Image Size \\
\hline SqueezeNet & 18 & 4.6 & 1.2 & $227 \times 227 \times 3$ \\
ResNet-18 & 18 & 44.0 & 11.7 & $224 \times 224 \times 3$ \\
ResNet-101 & 101 & 167.0 & 44.6 & $224 \times 224 \times 3$ \\
Inception-ResNet-V2 & 164 & 209.0 & 55.9 & $299 \times 299 \times 3$ \\
\hline
\end{tabular}




\subsubsection{Five-Fold Cross-Validation and Data Augmentation}

In the next step, five-fold cross-validation was performed. This method was used to overcome the deviation in small datasets used to train the deep-learning models for image classification (Figure 2). Dataset 1 (1000 images) was randomly divided into five groups of 200 images each. Four of these groups were used as training data, and the remaining group was used as validation data. It was carefully ensured that the same radiographs are not included in the training and validation data.

To prevent overfitting due to the small number of data samples, the amount of training data was increased through data augmentation. Training images were rotated from -7 to 7 , scaled horizontally and vertically from 0.9 to 1.1 , and translated horizontally and vertically from -5 to 5 pixels.

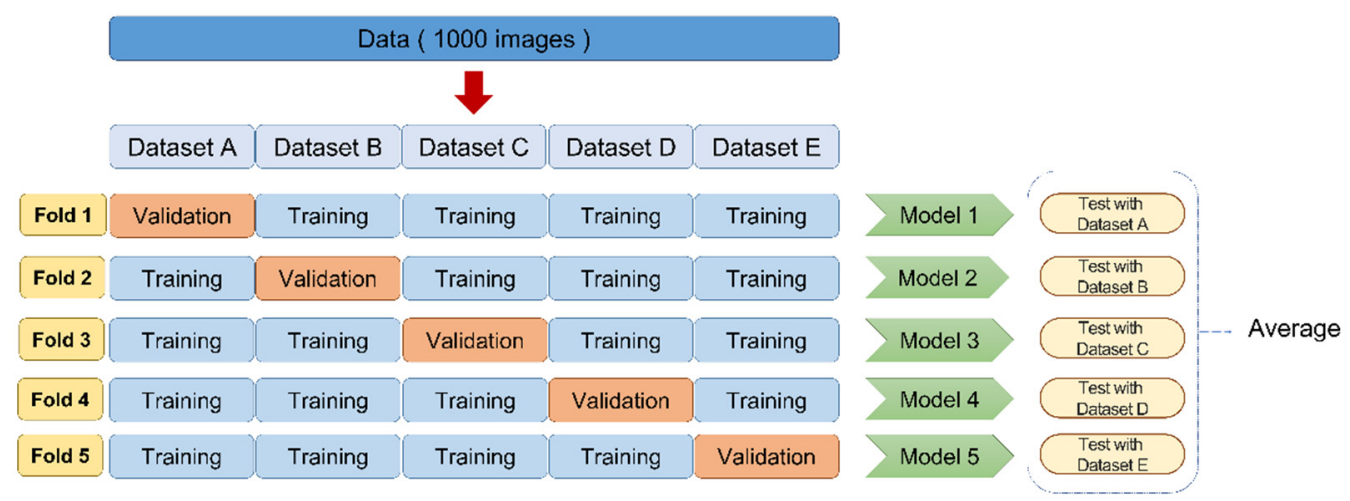

Figure 2. Five-fold cross-validation. The data were randomly divided into 5 groups, each consisting of 200 images. Four of these groups were used as training data and the remaining group was used as validation data. The diagnostic performance for each cross-validation set was evaluated, and the average of the five models was regarded as the estimated performance.

\subsubsection{Training Configuration}

An NVIDIA Titan RTX (i9-7980XE CP, 6 GB ram) was used for training the networks with MATLAB 2019b GPU (MathWorks, Natick, MA, USA). The models were trained for up to 500 epochs using the Adam optimizer. The size of the mini-batch was 16, and the initial learning rate was $10^{-4}$. The training process was stopped prematurely if the validation accuracy did not increase more than 30 times to avoid overfitting of the pretrained network.

\subsubsection{Diagnostic Performance Evaluation}

The diagnostic performance for each fold was calculated, and the average of the fivefold procedure was considered and evaluated as the final diagnostic performance of the deep-learning model for each network. The accuracy (1), precision (2), recall (3), F1-score (4), and area under the curve (AUC) values from the receiver operating characteristic (ROC) curve were used to evaluate the performance of the models.

$$
\begin{gathered}
\text { Accuracy }=\frac{T P+T N}{T P+T N+F P+F N} \\
\text { Precision }=\frac{T P}{T P+F P} \\
\text { Recall }=\frac{T P}{T P+F N} \\
\text { F1-score }=\mathbf{2} \times \frac{\text { Recall } \times \text { Precision }}{\text { Recall }+ \text { Precision }}
\end{gathered}
$$

$T P$ : true positive, $F P$ : false positive, $F N$ : false negative, and $T N$ : true negative. 


\subsubsection{Model Visualization}

Using the class activation map, the area of the image that affected the decision of AI was visualized and compared with the actual mesiodens location. A class activation map was generated for each network by obtaining the weighted sum of the last convolutional features (activation maps) using the fully connected layer weights [14].

\subsubsection{Comparison of the Ability of Deep-Learning Models and Human Groups}

To compare the abilities of the deep-learning models and human evaluators to classify mesiodens, 100 panoramic radiographs were classified in dataset 2 . As human evaluators, six pediatric dentists with more than five years of clinical experience and six general dentists with less than a year of clinical experience participated in the study. Human evaluators and four deep-learning models classified dataset 2 , and the times taken were measured. The accuracy, precision, recall, and F1-score for the classification results were calculated, as was the average value for each group.

\subsubsection{Statistical Analysis}

The investigated data were analyzed using SPSS 26.0 (SPSS Inc., IBM, Chicago, IL, USA). The Kruskal-Wallis test was used to verify the statistical significance of the classification performance of deep-learning models and human evaluators.

\section{Results}

\subsection{Classification Performance of Deep-Learning Models}

Table 3 details the mesiodens classification performance of the deep-learning models for dataset 1. SqueezeNet showed relatively lower accuracy, precision, and F1-score than those of the other three networks. ResNet-101 and Inception-ResNet-V2 showed evenly high values in all categories. The AUC values of the deep-learning models that delivered the best performance were 0.862 for SqueezeNet, 0.955 for ResNet-18, 0.941 for ResNet-101, and 0.932 for Inception-ResNet-V2 (Figure 3).

Table 3. Performances of deep-learning models on dataset 1.

\begin{tabular}{ccccc}
\hline Pre-Trained Network & Accuracy & Precision & Recall & F1-Score \\
\hline SqueezeNet & 0.833 & 0.779 & 0.960 & 0.855 \\
ResNet-18 & 0.914 & 0.883 & 0.958 & 0.918 \\
ResNet-101 & 0.927 & 0.911 & 0.948 & 0.928 \\
Inception-ResNet-V2 & 0.924 & 0.916 & 0.934 & 0.925 \\
\hline
\end{tabular}

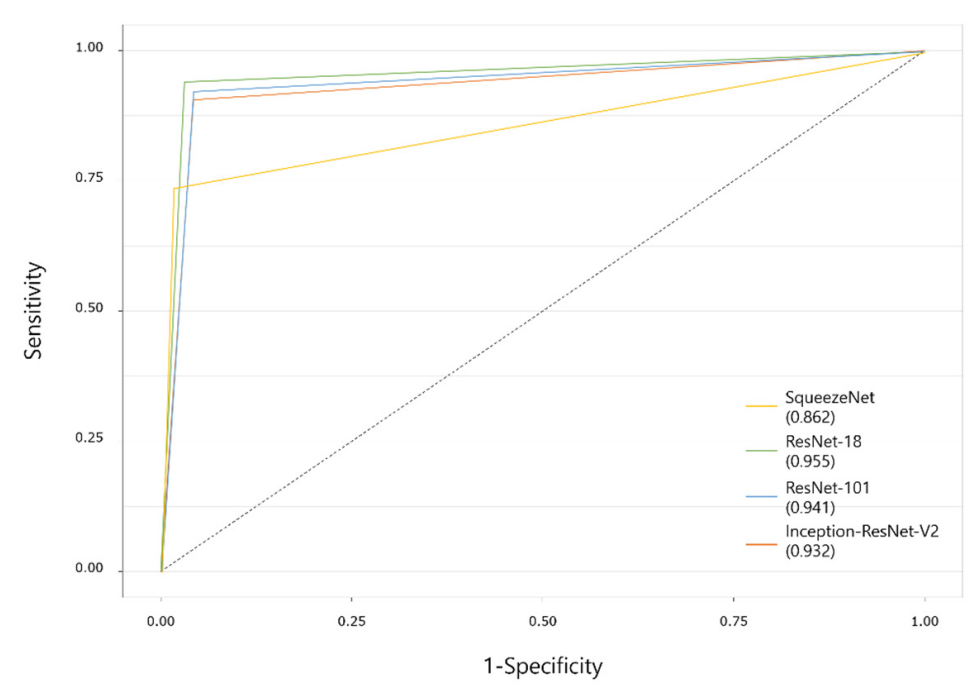

Figure 3. Receiver operating characteristic curves of deep-learning models on dataset 1 . Numbers in parentheses show the area under the curve values. 


\subsection{Visualization of Model Classification}

To identify the image regions that affect the classification result, we represented the regions with heat maps using class activation maps. The results of these heat maps for the classification are presented in Figure 4. In images with mesiodens, the deep-learning models focused on the actual locations of the mesiodens in many cases, but there was a slight difference in focus for each network. For images without mesiodens, the networks tended to evaluate the maxillary anterior region as a whole, including permanent incisors.

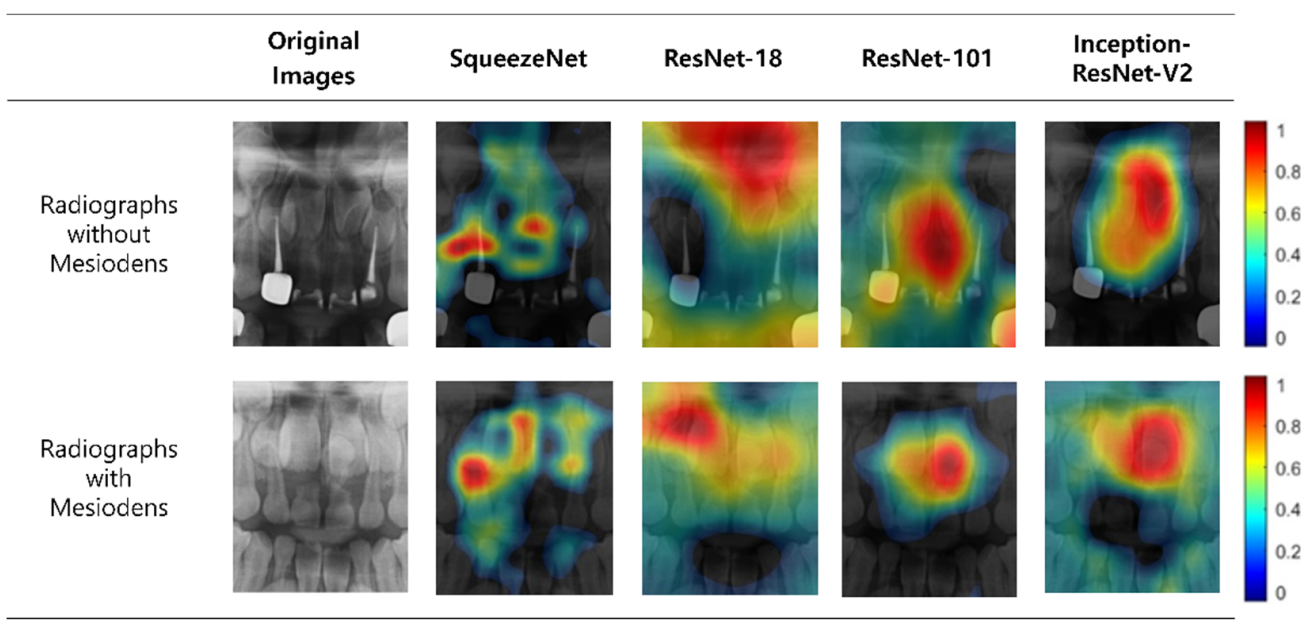

Figure 4. Example of the class activation maps of four deep-learning models.

\subsection{Comparative Evaluation of the Abilities of the Deep-Learning Models and Human Groups}

The classification results of the human evaluators and the deep-learning models on dataset 2 are presented in Table 4. The results of the Kruskal-Wallis test in terms of the accuracy, recall, and F1-score of the three groups of general dentists, pediatric dentists, and deep-learning models showed significant differences $(p<0.05$, Table 4 , Figure 5). Figure 6 shows the correct answer for each question according to the interpreter. The lists of incorrectly diagnosed problems tended to be similar among the deep-learning models, whereas the similarity decreased between human evaluators and deep-learning models.
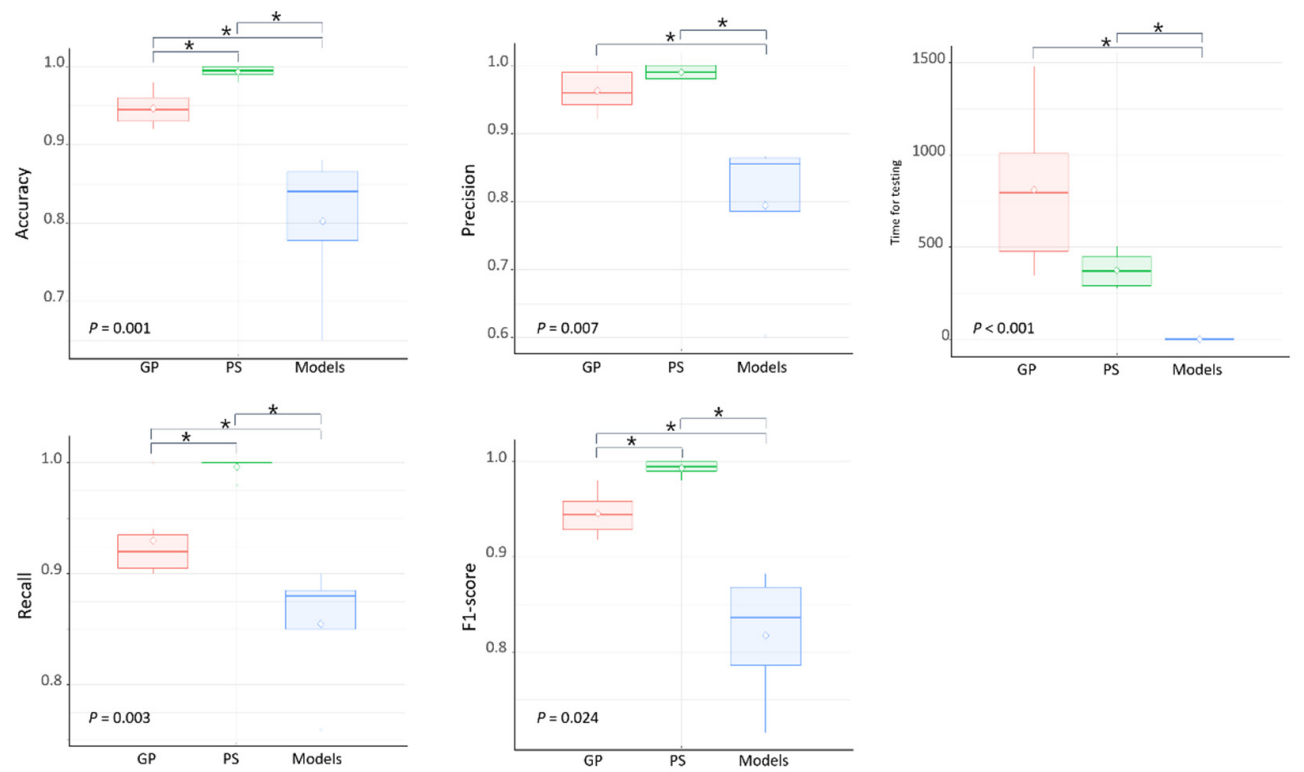

Figure 5. Boxplot of diagnostic performances among three groups. Kruskal-Wallis test was performed to analyze the statistical significance of specificity. GP: General Practitioners, PS: Pediatric Specialists, ${ }^{*} p<0.05$; Kruskal-Wallis test. 
Table 4. Comparison of diagnostic performances of deep-learning models and human groups.

\begin{tabular}{cccccc}
\hline & Accuracy & Precision & Recall & F1-Score & Time for Testing (s) \\
\hline GP & 0.95 & 0.96 & 0.90 & 0.93 & $811.8 \pm 426.1$ \\
PS & 0.99 & 0.99 & 1.00 & 0.93 & $375.5 \pm 95.9$ \\
SqueezeNet & 0.65 & 0.60 & 0.88 & 0.72 & $1.5 \pm 1.4$ \\
ResNet-18 & 0.82 & 0.86 & 0.76 & 0.81 & \\
ResNet-101 & 0.86 & 0.85 & 0.88 & 0.86 & \\
Inception-ResNet-V2 & 0.88 & 0.87 & 0.90 & 0.88 & \\
\hline
\end{tabular}

GP: General Practitioners, PS: Pediatric Specialists.

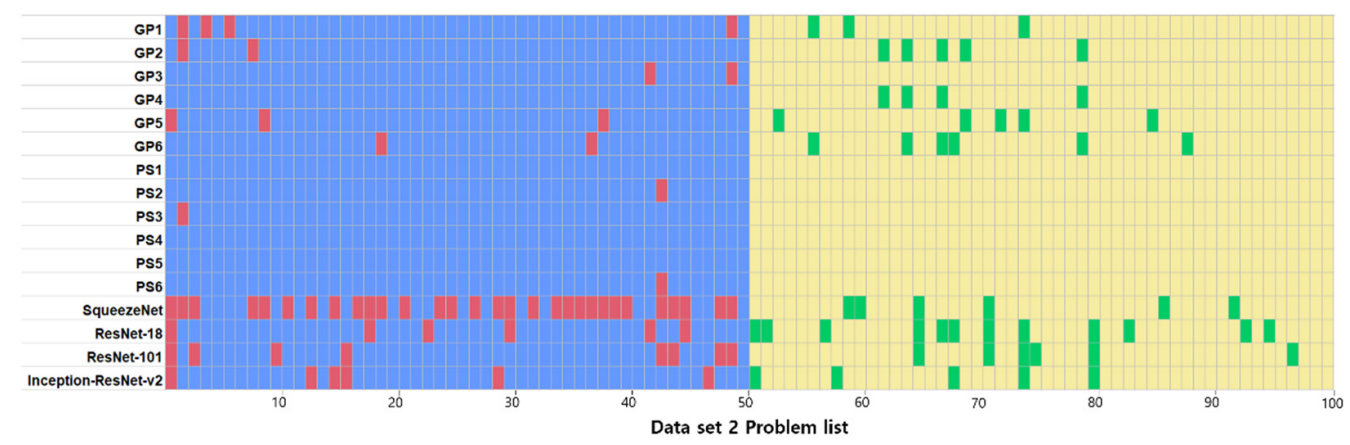

Figure 6. Differences in answers between human evaluators and deep-learning models on dataset 2. Blue: true positive, Red: false negative, Yellow: true negative, and Green: false positive. GP: General Practitioners, PS: Pediatric Specialists.

\section{Discussion}

In 2017, Anthonappa et al. [8] conducted a study on the effectiveness of panoramic radiographs in identifying supernumerary teeth. According to that study, the sensitivity of supernumerary teeth classification using panoramic radiographs was 0.50 . Further, a significant difference in sensitivity was confirmed between pediatric dentists $(0.60)$ and dentists with less than one year of experience (0.39). Based on these results, the authors stated that the clinical experience of the dentist significantly influences the identification of supernumerary teeth with panoramic radiographs and that it is difficult to trust panoramic radiographs as a tool for identifying supernumerary teeth.

Recently, research on deep-learning systems has been increasing in various fields, including oral and maxillofacial radiology. Among the various deep-learning functions, the classification function is frequently used in panoramic radiographs and has been reported to perform excellently in the evaluation of various diseases [9,11-13]. Herein, we attempted to provide a background that can help clinicians in realizing accurate and rapid diagnosis by creating models that automatically classify mesiodens in panoramic radiographs using CNNs.

In this study, four popular pre-trained networks in Pareto frontier were applied for mesiodens classification while considering the accuracy and computational burden. Pareto frontier in the field of deep learning comprises networks that outperform other networks in terms of accuracy and prediction time [18]. Deeper networks can generally achieve higher accuracies by learning richer feature representations. However, deep networks require larger amounts of computing power and are characterized by longer prediction times when using graphic processing units (GPUs), and these are difficult to realize in average research and clinical environments. Therefore, in this study, we found the optimal pre-trained network architecture that satisfies the requirements of both accuracy and computing power for the classification of mesiodens on panoramic radiographs. The tested networks were SqueezeNet, ResNet-18, ResNet-101, and Inception-ResNet-V2.

All the deep-learning models used in this study yielded AUC values exceeding 0.85 on dataset 1 . Among the four models, SqueezeNet, which has the shallowest depth and parameters, had a relatively low accuracy of 0.833 . ResNet-101 and Inception-ResNetV2, which are relatively deep networks, deliver the highest performance in most cases, 
although there are some differences depending on the metrics. In this study, the number and depth of parameters of the $\mathrm{CNN}$ and the accuracy of the model showed positive correlations, supporting the fact that the deeper the network, the higher the accuracy [19].

The CNN had the disadvantage of not being able to explain the characteristics of machine learning and decisions made by learning owing to the black-box effect [20]. McNamara et al. [21] reported that the class activation map helps solve the black-box effect by visualizing the area observed by the $\mathrm{CNN}$ model during object classification, and various attempts have been made using class activation maps since then in this regard. In this study, the part that plays an important role in classifying mesiodens in the panoramic radiograph was identified, using the class activation map. In most images, the network observed the actual locations of the mesiodens, but there was a slight difference in focus between all the networks. If a deep-learning model is visualized using class activation maps, it will influence humans to trust the decision made by the AI. It is possible to increase the clinical applicability of the deep-learning models by visualizing the presence and locations of the excessive number of teeth.

In this study, when the classification abilities of humans and deep-learning models were compared, the accuracy of the deep-learning models was lower than that of the humans, but the detection was significantly faster. It took an average of $811.8 \mathrm{~s}$ for the general dentist group and $375.5 \mathrm{~s}$ for the pediatric dentist group to perform evaluations on dataset 2, but the deep-learning models performed detection and classification of the entire test set within a few seconds, showing a significant difference. In a study conducted by Hiraiwa et al. [12] in 2019, the model using AlexNet and GoogleNet completed the classification of the distal root of the mandibular first molar in 163 panoramic radiographs within 9 and $11 \mathrm{~s}$, with accuracies of $87.4 \%$ and $85.3 \%$, respectively. If a deep-learning model is used as an auxiliary means for diagnosing mesiodens after taking panoramic radiographs, it would help shorten the diagnosis time of clinicians with little experience with mixed dentition panoramic radiographs. In addition, it will be possible to reduce complications due to mesiodens by increasing the probability of detecting mesiodens in advance.

In this study, the accuracies of the deep-learning models were slightly lower on dataset 1 than on new data (dataset 2) that were not used for validation. This is thought to be due to overfitting with a small number of training data. The performance of deeplearning systems typically depends on the amount of data available for training [22,23]. There was a limitation in collecting the training data because this study incorporated panoramic radiographs from a single institution. In a follow-up study, it is thought that the performance of the model can be further improved by training the network using radiographs from multiple institutions to develop a larger dataset.

This study also had some other limitations. Firstly, the dental age, which affects the difficulty of detecting mesiodens, of both the experimental group and the control group were set under the same conditions. As a result of this, the experimental group and the control group had slight differences in age and gender of the subjects. Secondly, it was difficult to determine the numbers or exact locations of mesiodens by only classifying their presence or absence in panoramic radiographs. The use of a network including object detection and classification functions, such as Single Shot MultiBox Detector, can help identify the distribution of supernumerary teeth. Lastly, in pediatric patients with primary dentition, intraoral radiographs are often taken instead of panoramic radiographs to minimize unnecessary radiation dose. A follow-up study using intraoral radiographs of the maxillary anterior region for an early detection of supernumerary teeth is necessary.

\section{Conclusions}

The deep-learning network models used in this study delivered high accuracy in classifying the presence of mesiodens in the mixed dentition panoramic radiographs. Although there was a difference in focus on class activation maps between the networks, the actual locations of the mesiodens and their surrounding teeth were typically observed. 
The results of this study are expected to help clinicians make decisions by automatically classifying these teeth on panoramic radiographs taken during the eruption period of the maxillary anterior teeth, thereby reducing complications that may arise from failure of early detection of mesiodens.

Author Contributions: Conceptualization, Y.A., J.J.H. and J.S.; methodology, J.J.H.; formal analysis, Y.A., J.J.H. and Y.-H.J.; investigation, Y.A.; writing-original draft preparation, Y.A.; writing-review and editing, J.J.H., Y.-H.J., T.J. and J.S.; visualization, Y.A.; supervision, T.J.; project administration, J.S.; funding acquisition, J.J.H. All authors have read and agreed to the published version of the manuscript.

Funding: This research was supported by a grant of the Korea Health Technology R\&D Project through the Korea Health Industry Development Institute (KHIDI), funded by the Ministry of Health and Welfare, Republic of Korea (grant number: HI19C0824).

Institutional Review Board Statement: The study was conducted according to the guidelines of the Declaration of Helsinki, and approved by the Institutional Review Board of the Pusan National University Dental Hospital (approval number: PNUDH-2020-006, approval date: 25 March 2020).

Informed Consent Statement: By the Institutional Review Board of Pusan National University Dental Hospital, patient consent was waived for the need for individual informed consent as this study had a non-interventional retrospective design and all the data were analyzed anonymously; therefore, no written/verbal informed consent was obtained from the participants.

Data Availability Statement: Restrictions apply to the availability of these data. Data used in this study were obtained from Pusan National University Dental Hospital and are available with the permission of the Institutional Review Board of Pusan National University Dental Hospital, Pusan National University, Dental Research Institute.

Conflicts of Interest: The authors declare no conflict of interest.

\section{References}

1. Rajab, L.; Hamdan, M. Supernumerary teeth: Review of the literature and a survey of 152 cases. Int. J. Paediatr. Dent. 2002, 12, 244-254. [CrossRef]

2. Mallineni, S.K. Supernumerary teeth: Review of the literature with recent updates. Conf. Papers Sci. 2014. [CrossRef]

3. Meighani, G.; Pakdaman, A. Diagnosis and management of supernumerary (mesiodens): A review of the literature. J. Dent. (Tehran) 2010, 7, 41.

4. Katheria, B.C.; Kau, C.H.; Tate, R.; Chen, J.W.; English, J.; Bouquot, J. Effectiveness of impacted and supernumerary tooth diagnosis from traditional radiography versus cone beam computed tomography. Pediatr. Dent. 2010, 32, 304-309.

5. Brenner, D.J.; Elliston, C.D.; Hall, E.J.; Berdon, W.E. Estimated risks of radiation-induced fatal cancer from pediatric CT. Am. J. Roentgenol. 2001, 176, 289-296. [CrossRef] [PubMed]

6. Fanning, B. CBCT-the justification process, audit and review of the recent literature. J. Ir. Dent. Assoc. 2011, 57, 256-261. [PubMed]

7. Han, W.J.; Kim, J.S. Effective Dose from Cone Beam Ct for Imaging of Mesiodens. J. Korean Acad. Pediatr. Dent. 2012, 39, 273-279. [CrossRef]

8. Anthonappa, R.P.; King, N.M.; Rabie, A.B.M.; Mallineni, S.K. Reliability of panoramic radiographs for identifying supernumerary teeth in children. Int. J. Paediatr. Dent. 2012, 22, 37-43. [CrossRef] [PubMed]

9. Ohashi, Y.; Ariji, Y.; Katsumata, A.; Fujita, H.; Nakayama, M.; Fukuda, M.; Nozawa, M.; Ariji, E. Utilization of computer-aided detection system in diagnosing unilateral maxillary sinusitis on panoramic radiographs. Dentomaxillofac. Radiol. 2016, 45, 20150419. [CrossRef] [PubMed]

10. Lee, J.H.; Kim, D.H.; Jeong, S.N.; Choi, S.H. Detection and diagnosis of dental caries using a deep learning-based convolutional neural network algorithm. J. Dent. 2018, 77, 106-111. [CrossRef]

11. Murata, M.; Ariji, Y.; Ohashi, Y.; Kawai, T.; Fukuda, M.; Funakoshi, T.; Kise, Y.; Nozawa, M.; Katsumata, A.; Fujita, H. Deeplearning classification using convolutional neural network for evaluation of maxillary sinusitis on panoramic radiography. Oral Radiol. 2019, 35, 301-307. [CrossRef]

12. Hiraiwa, T.; Ariji, Y.; Fukuda, M.; Kise, Y.; Nakata, K.; Katsumata, A.; Fujita, H.; Ariji, E. A deep-learning artificial intelligence system for assessment of root morphology of the mandibular first molar on panoramic radiography. Dentomaxillofac. Radiol. 2019, 48, 20180218. [CrossRef] [PubMed]

13. Yang, H.; Jo, E.; Kim, H.J.; Cha, I.H.; Jung, Y.S.; Nam, W.; Kim, J.Y.; Kim, J.K.; Kim, Y.H.; Oh, T.G. Deep learning for automated detection of cyst and tumors of the jaw in panoramic radiographs. J. Clin. Med. 2020, 9, 1839. [CrossRef]

14. Zhou, B.; Khosla, A.; Lapedriza, A.; Oliva, A.; Torralba, A. Learning deep features for discriminative localization. In Proceedings of the IEEE Conference on Computer Vision and Pattern Recognition, Las Vegas, NV, USA, 27-30 June 2016; pp. $2921-2929$. 
15. Kuwada, C.; Ariji, Y.; Fukuda, M.; Kise, Y.; Fujita, H.; Katsumata, A.; Ariji, E. Deep learning systems for detecting and classifying the presence of impacted supernumerary teeth in the maxillary incisor region on panoramic radiographs. Oral Surg. Oral Med. Oral Pathol. Oral Radiol. 2020, 130, 464-469. [CrossRef]

16. Seung, J.; Kim, J.; Yang, Y.; Lim, H.; Le, V.N.T.; Lee, D. Identification of Mesiodens Using Machine Learning Application in Panoramic Images. J. Korean Acad. Pediatr. Dent. 2021, 48, 221-228.

17. Hellman, M. An introduction to growth of the human face from infancy to adulthood. Int. J. Orthod. Oral Surg. Radiogr. 1932, 18, 777-798. [CrossRef]

18. Bianco, S.; Cadene, R.; Celona, L.; Napoletano, P. Benchmark analysis of representative deep neural network architectures. IEEE Access 2018, 6, 64270-64277. [CrossRef]

19. Zhong, G.; Ling, X.; Wang, L.N. From shallow feature learning to deep learning: Benefits from the width and depth of deep architectures. Wiley Interdiscip. Rev. Data Min. Knowl. Discov. 2019, 9, e1255. [CrossRef]

20. Mahdi, F.P.; Motoki, K.; Kobashi, S. Optimization technique combined with deep learning method for teeth recognition in dental panoramic radiographs. Sci. Rep. 2020, 10, 1-12. [CrossRef]

21. McNamara, J.A., Jr.; Franchi, L. The cervical vertebral maturation method: A user's guide. Angle Orthod. 2018, 88, 133-143. [CrossRef] [PubMed]

22. Cha, D.; Pae, C.; Seong, S.B.; Choi, J.Y.; Park, H.J. Automated diagnosis of ear disease using ensemble deep learning with a big otoendoscopy image database. EBioMedicine 2019, 45, 606-614. [CrossRef] [PubMed]

23. Fukuda, M.; Inamoto, K.; Shibata, N.; Ariji, Y.; Yanashita, Y.; Kutsuna, S.; Nakata, K.; Katsumata, A.; Fujita, H.; Ariji, E. Evaluation of an artificial intelligence system for detecting vertical root fracture on panoramic radiography. Oral Radiol. 2019, 36, 337-343. [CrossRef] [PubMed] 